\title{
New route for preparation of luminescent mercaptoethanoate capped cadmium selenide quantum dots
}

\author{
MANOJ E WANKHEDE, SHAUKATALI N INAMDAR, APARNA DESHPANDE ${ }^{\dagger}$, \\ ANIKET R THETE, RENU PASRICHA ${ }^{\dagger \dagger}$, SULABHA K KULKARNI ${ }^{\dagger}$ and \\ SANTOSH K HARAM* \\ Department of Chemistry, ${ }^{\dagger}$ Department of Physics, DST Centre for Nanoscience, University of Pune, \\ Pune 411 007, India \\ ${ }^{\dagger \dagger}$ National Chemical Laboratory, Pune 411 008, India
}

\begin{abstract}
We report a synthesis of cadmium selenide quantum dots (Q-CdSe) by refluxing a mixture of cadmium acetate, selenium powder, sodium sulfite and 2-mercaptoethanol in $\mathbf{N}, \mathrm{N}^{\prime}$-dimethyl formamide (DMF)/water solution. X-ray and electron diffractions suggest the formation of hexagonal phase of size quantized CdSe. Based on TEM analysis, the formation of nanoparticles with an average diameter of $3.5 \pm 0.5 \mathrm{~nm}$ is inferred. Their sols in DMF and dimethyl sulphoxide (DMSO) gave characteristic absorption peaks at $300 \mathrm{~nm}$ and $327 \mathrm{~nm}$, which is attributed to the formation of high quality, size quantized CdSe particles. Extracted particles from the sol were readily redispersed in DMF and DMSO, which were diluted further with water without losing their optical and colloidal properties. FTIR spectroscopy suggested the formation of 2mercaptoethanol thiolate on the particle surface, with free -OH groups available for linkage. Sols in DMSO and their solutions in water displayed an intense photoluminescence (PL).
\end{abstract}

Keywords. Semiconductor quantum dots; chalcogenides; chemical synthesis; photoluminescence.

\section{Introduction}

Given the fact that the fluorescence properties of the semiconductor quantum dots (QDs) can be tuned by controlling the size, their use as a fluorescence label to biological macromolecules has attracted a considerable attention in recent years (Bruchez et al 1998; Chan and Nie 1998; Mattoussi et al 2001; Pathak et al 2001; Chen and Rosenzweig 2002, 2003; Parak et al 2002). These size tunable properties allow one to choose an emission wavelength suitable for particular biological system and to synthesize QDs based probe of required materials and size. Unlike conventional organic probes, nanoparticles exhibit narrow symmetric emission peak. Besides, these have a large absorption cross-section due to large density of states and overlapped band structure. Conventional probes on the other hand have narrow absorption maxima. Therefore, these properties allow the excitation of multiple QDs probes with a single light source. All these properties make QDs as a desirable fluorescent probe. Preparation of QDs in predefined particle size, shape and having narrow size distribution in size, however, have always been a challenging and yet to be achieved goal.

Among various semiconductors investigated to date, II-VI materials, especially CdSe quantum dots (Q-CdSe),

\footnotetext{
*Author for correspondence (haram@chem. unipune.ernet.in)
}

have shown high quantum yield and size dependent narrow emissions in entire visible spectrum thus viewed as potential material in the bio-labeling applications. Various methods have been reported for the preparation of Q-CdSe, which can be classified based on precursors, nature of capping agents and dispersing media. An organometallic route introduced by Bawendi and coworkers (Murray et al 1993), which consisted of reaction of a dimethyl cadmium with tri- $n$-octylphosphine selenide in tri- $n$-octyl phosphine oxide (TOPO) at ca. $300^{\circ} \mathrm{C}$ have been the most extensively tried route. Subsequently, lot of variants of this procedure have been reported with a variation in the nature of precursors, medium and temperature (Bowen-Katari et al 1994; Trindade and O'Brien 1996; Trindade et al 1997; Xie et al 1999; Gautam et al 2001; Hu et al 2001). Among them, in a method developed by Peng and Peng (2001), gaseous and poisonous dimethyl cadmium in the earlier methods was replaced by more safer-to-handle $\mathrm{CdO}$ solid. In general, TOPO capped Q-CdSe are very stable against flocculation and isolable as a solid powder. Chemisorption of TOPO passivates particle surface effectively. Thus, photoluminescence (PL) is ascribed to band-to-band transitions, which can be tuned by controlling the size. These particles in native form, however, are not biocompatible due to lack in appropriate functional groups like $-\mathrm{OH}$, $-\mathrm{NH}_{2}$ or $-\mathrm{COOH}$ which may interact with biomolecules. Water insolubility due to hydrophobic TOPO cap is yet another limitation. Its water solubility has been improved 
with a limited success by (i) replacing TOPO cap with bifunctional ligands like mercaptoacetic acid or mercaptoamines by a ligand exchange method, (ii) capping native TOPO capped particles further with octylamine modified polyacrylic acid and (iii) forming silica shell on the native particles.

Therefore, it sounds more profitable to directly prepare Q-CdSe in hydrosols form. Synthetic procedures to prepare them in general consisted of reaction of cadmium salts with $\mathrm{H}_{2} \mathrm{Se}$ (Tian et al 1996; Trindade et al 2001), $\mathrm{N}, \mathrm{N}^{\prime}$-dimethyl selenourea (Rogach et al 2000; Lin et al 2005) or $\mathrm{Na}_{2} \mathrm{SeSO}_{3}$ (Ma et al 2002) in presence of capping agents or surfactants. Its limitations, however, have been poor control over size and shape. Isolation and processing of these particles have been less feasible due to the poor redispersity. Moreover, water based procedures have been found to give the particles with various surface defects including oxide layers, which lead to poor and unpredictable PL properties (Rogach et al 1999; Aldana et al 2001; Vasiliev et al 2004). Therefore, it will still be challenging to produce monodispersed Q-CdSe having (i) good fluorescence properties, (ii) solubility in water and (iii) having a functional group which can be used to anchor the particles to the biomolecules.

With this motivation, we tried to develop a convenient and safe, one-pot route for the synthesis of 2-mercaptoethanol capped Q-CdSe, which were isolated and redispersed in water-based solvents, without losing any of its optical properties. The method neither demands high temperature nor an inert atmosphere, thus can be reproduced in common laboratory. The free $-\mathrm{OH}$ group present on 2-mercaptoethanol cap makes these particles more attractive as it can be used to anchor the particles to the biological moiety. Thus, these particles can be viewed as a potential candidate as a fluorescent probe in bioimaging applications.

\section{Experimental}

\subsection{Materials}

Cadmium acetate dihydrate (Merck), Se powder (S.D. Fine Chemicals, India), sodium sulfite anhydrous (Merck), 2-mercaptoethanol (S.D. Fine Chemicals, India), and all the other chemicals were of analytical reagent grade and used as received without any further purification.

\subsection{Method of preparation}

$0.587 \mathrm{~g}$ Cadmium acetate was transferred to $250 \mathrm{ml}$ threenecked round bottom flask containing $25 \mathrm{ml}$ distilled water. The solution was stirred till its complete dissolution. $85 \mathrm{ml} \mathrm{DMF}$ and $0.5 \mathrm{ml} \mathrm{2-mercaptoethanol} \mathrm{were} \mathrm{added}$ into it. After stirring for an hour, $0.130 \mathrm{~g}$ of $\mathrm{Na}_{2} \mathrm{SO}_{3}$ was added. The solution was stirred for $15 \mathrm{~min}$ and refluxed further for about $90 \mathrm{~min}$. $0.025 \mathrm{~g}$ Se powder was added into it and refluxed further for $3 \mathrm{~h}$. The effective molar concentration of $\mathrm{Cd}$ acetate, $\mathrm{Se}, \mathrm{Na}_{2} \mathrm{SO}_{3}$ and 2-mercaptoethanol in bath were $2.2,0.32,1.03$ and $5.74 \mathrm{mM}$, respectively. After cooling the bath, formation of pale yellow solution with unreacted Se settled at the bottom of flask was observed. Se was separated by simple filtration. The sol was concentrated in rotary evaporator under vacuum. The acetone was added into it as antisolvent which led to a flocculation of the particles. It was then centrifuged at $5000 \mathrm{rpm}$ for complete separation of solid. The supernatant liquid was decanted and the solid was washed 3-4 times with copious amount of methanol. The resultant precipitate was dried and stored under vacuum till further analysis.

\subsection{Characterization}

Powder X-ray diffractograms (XRD) on the dried precipitate were recorded using a JEOL JDX-8030 X-ray diffractometer having $\mathrm{CuK} \alpha$ source (40 kV and $20 \mathrm{~mA}$ ). Samples were prepared by sprinkling the precipitate over glass slides. The diffractograms were recorded between angles $20^{\circ}$ and $80^{\circ}$ with a scan rate of $2 \mathrm{~s}$ per step $\left(0.05^{\circ}\right.$ per step $)$. For the routine characterization and reaction kinetics studies, UV-vis (Shimadzu UV-1650PC) spectra were recorded at the stipulated time intervals. Steady state photoluminescence (PL) spectra were recorded at room temperature using a Shimadzu RF-5301PC spectrofluorometer. IR spectroscopic measurements were carried out using Shimadzu FTIR-8400 spectrophotometer. Typically ca. $10 \mathrm{mg}$ of the dried precipitate was mixed with ca. $100 \mathrm{mg}$ of spectroscopic-grade potassium bromide, and the resultant mixture was ground to a fine powder by using a mortar and pestle. A portion of the mixture was pressed into a transparent homogeneous pellet at 20000 psi. For liquid samples, sodium chloride window cell was used.

To study the morphology of the particles, transmission electron micrographs (TEM) were recorded using a Philips CM200 transmission electron microscope. A drop of the freshly prepared sol was dried on carbon-coated TEM grid. The measurements were carried out at $88 \mathrm{kV}$.

\section{Results and discussion}

\subsection{Formation of Q-CdSe}

Cadmium ions are known to react with sodiumselenosulfite to form $\mathrm{CdSe}$. The reaction is believed to proceed by two plausible steps (Ma et al 2002),

$$
\mathrm{Na}_{2} \mathrm{SO}_{3}+\mathrm{Se} \rightarrow \mathrm{Na}_{2} \mathrm{SeSO}_{3},
$$

followed by its reaction with $\mathrm{Cd}^{2+}$ ions by the mechanism 


$$
\mathrm{Cd}^{2+}+\mathrm{SeSO}_{3}^{2-}+2 \mathrm{OH}^{-} \rightarrow \mathrm{CdSe}+\mathrm{SO}_{4}^{2-}+\mathrm{H}_{2} \mathrm{O} .
$$

Absorption spectrum recorded on as-prepared sol in DMF-water mixture is depicted in figure 1 (a). Observed sharp peak at $327 \mathrm{~nm}$ and shoulder at $300 \mathrm{~nm}$ are comparable with the one reported for TOPO capped Q-CdSe sols (Murray et al 1993). These transitions have been assigned to the $S_{1} \leftarrow S_{0}$ and $S_{2} \leftarrow S_{0}$, of size quantized particles. Separation of these transitions in the form of two peaks has been associated to the narrow size distribution of the Q-CdSe $(3.5 \pm 0.5 \mathrm{~nm})$ particles (Gaponik et al 2002). To remove the unreacted precursors and other byproducts from the sol, it was flocculated, washed and vacuum dried. The dry powder was readily redispersed into polar solvents like DMF and DMSO. One such absorption spectrum for Q-CdSe sol in DMSO is as shown in figure 1(b), which is quite comparable to the native sol (refer figure 1(a)). The background absorption from unreacted precursors and byproducts was seen to decrease in the purified samples.

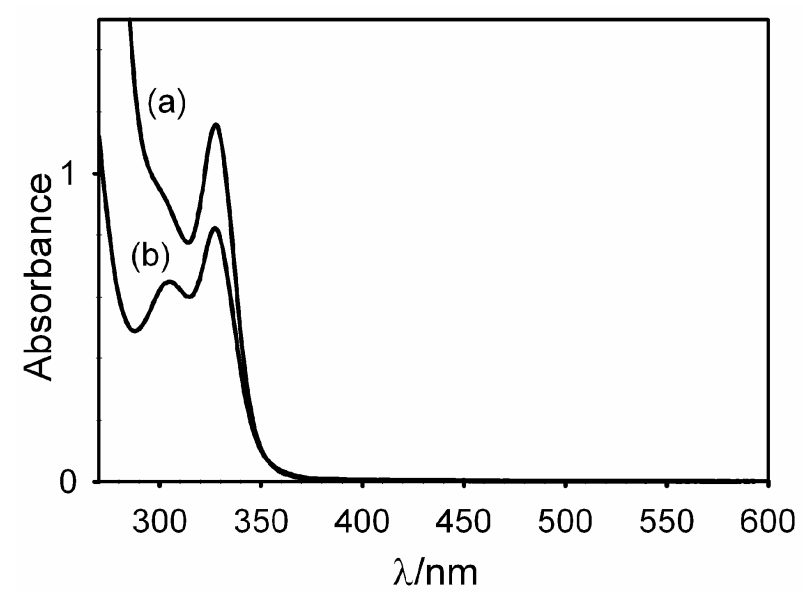

Figure 1. UV-vis spectra recorded on (a) as prepared sol and (b) after extraction and re-dispersion in DMSO.

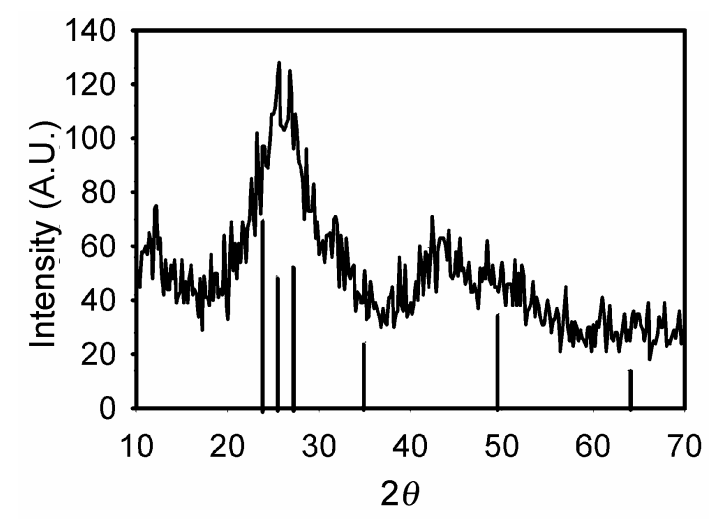

Figure 2. X-ray powder diffractogram (XRD) recorded on extracted Q-CdSe powder. The vertical bars are the ones reported for hexagonal phase of CdSe (JCPDS file number 8459).
Thus, the particles are processable without losing their optical properties.

One of the important properties of these sols was their redispersibility in the water based solvents too. The dry powder of sol was not freely redispersed in the distilled water. Nevertheless their sol in DMSO was diluted freely further with water, without deteriorating its optical or colloidal properties. It was attributed to the presence of a polar-OH group from 2-mercaptoethanol cap.

Figure 2 depicts X-ray powder diffractogram (XRD) recorded on the dried extracted sol. Broad peaks of the diffractogram have been attributed to the extremely small

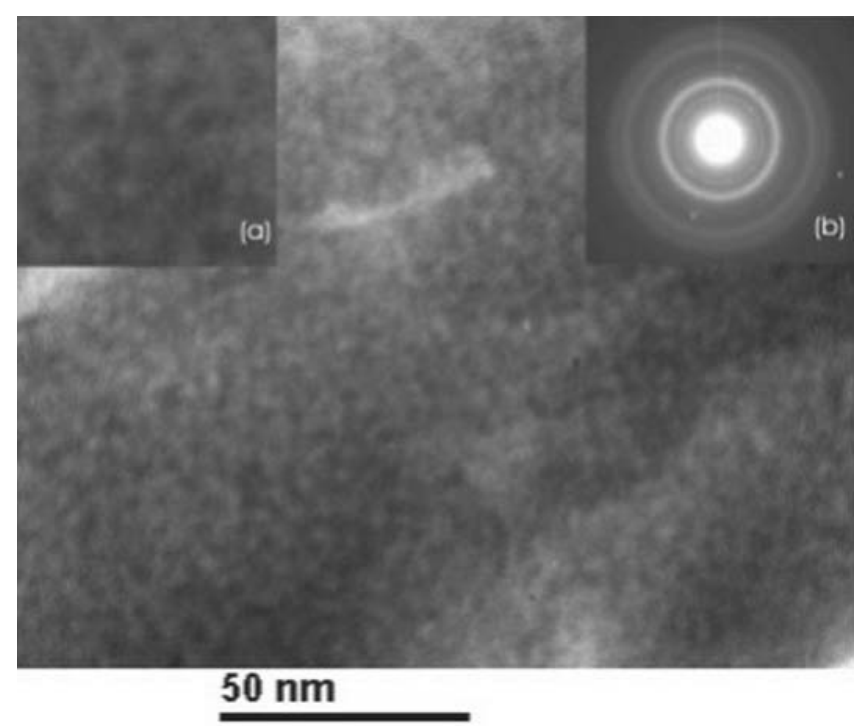

Figure 3. Low resolution TEM image recorded for CdSe nanoparticles. The digitally enlarged view of the micrograph is shown in inset (a). Inset (b) depicts selected area electron diffraction.

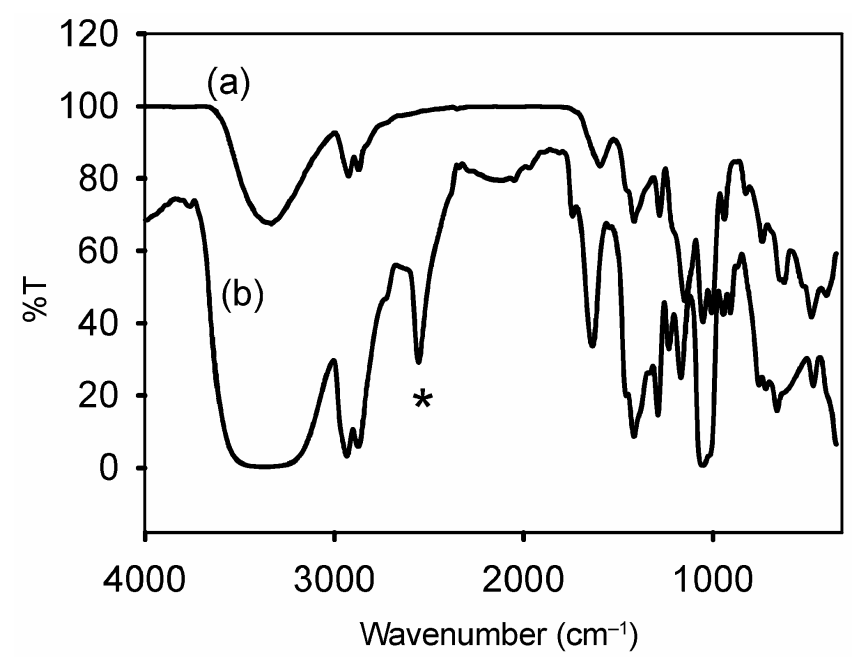

Figure 4. FTIR spectra recorded for (a) $\mathrm{KBr}$ pellet of extracted sol and (b) pristine 2-mercaptoethanol. * indicates the position for $\mathrm{S}-\mathrm{H}$ stretching band. 
grain size. The observed peaks were best fitted in the hexagonal phase of CdSe (JCPDS File 8-459). Due to extremely small grain size and overlapping of the peaks, it was not possible to apply the Scherrer equation to estimate the average particle size using XRD. Interestingly, a formation of traces of CdSTe have been reported along with Q-CdTe when the aqueous solution of $\mathrm{Cd}$ and $\mathrm{Te}$ was refluxed in presence of alkyl thiol (Gaponik et al 2002). This was attributed to the hydrolysis of thiol due to prolonged heating. The XRD recorded on our samples (refer figure 2), however, did not suggest the presence of CdSSe along with Q-CdSe. It could be due to the shorter heating duration, compared to the one reported in case of Q-CdTe (Gaponik et al 2002).

A typical low resolution TEM image obtained on dropcasted TEM grid is depicted in figure 3. The particles are seen to be uniformly distributed. From the available resolution, the average value for the size was found to be $3.5 \pm 0.5 \mathrm{~nm}$. An inset (b) in figure 3 depicts selected area electron diffractogram. The corresponding analysis is given in table 1, which also fitted faithfully in the hexagonal phase of CdSe (JCPDS File 8-459).

\subsection{FTIR analysis}

An adsorption of 2-mercaptoethanol on the particle surface was studied with the help of FTIR spectroscopy. The

Table 1. ED analysis.

\begin{tabular}{|c|c|c|}
\hline $\begin{array}{l}d \text { values from } \\
\text { JCPDS file }\end{array}$ & $d$ values from ED & $(h k l)$ \\
\hline $3 \cdot 720$ & $3 \cdot 828$ & $\left(\begin{array}{lll}1 & 0 & 0\end{array}\right)$ \\
\hline $3 \cdot 290$ & $3 \cdot 141$ & $\left(\begin{array}{lll}1 & 0 & 1\end{array}\right)$ \\
\hline $2 \cdot 151$ & $2 \cdot 213$ & $\left(\begin{array}{lll}1 & 1 & 0\end{array}\right)$ \\
\hline $1 \cdot 834$ & $1 \cdot 842$ & $\left(\begin{array}{lll}1 & 1 & 2\end{array}\right)$ \\
\hline 1.456 & 1.545 & $\left(\begin{array}{lll}2 & 0 & 3\end{array}\right)$ \\
\hline
\end{tabular}

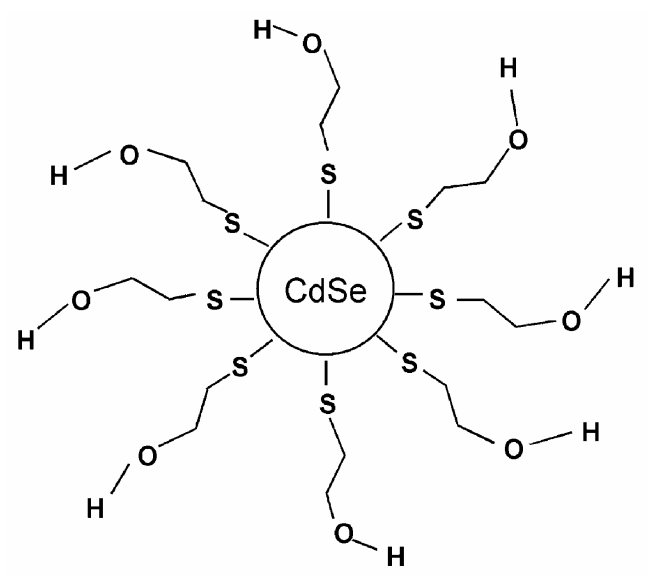

Figure 5. A sketch for the Q-CdSe capped with 2-mercaptoethanoate based on FTIR results. sample for this purpose was washed further several times with a copious amount of methanol and vacuum dried to remove physically adsorbed thiols. One such representative spectrum recorded on dried powder in the $\mathrm{KBr}$ pellet is shown in figure 4(a). For comparison, a spectrum for pristine 2-mercaptoethanol is overlayed in the plot (refer, figure 4(b)). The correlation between these spectra is listed in table 2. Majority of the peaks of pristine 2-mercaptoethanol matched with the peaks of 2-mercaptoethanol associated to Q-CdSe. The sharp peak (marked as * in figure 4) for -SH stretching was observed at $2563 \mathrm{~cm}^{-1}$ in case of pristine mercaptoethanol. It completely disappeared in Q-CdSe, which was attributed to the breaking of - $\mathrm{SH}$ bond (Hostetler et al 1996) while adsorption of alkyl thiolate group took place on the surface. $-\mathrm{OH}$ stretching and $-\mathrm{CH}_{2}$ bending vibrations in the range $3400 \mathrm{~cm}^{-1}$ and $1423 \mathrm{~cm}^{-1}$, respectively were observed in both the cases (Zhang and Wang 2003). From that, it is inferred that the $-\mathrm{OH}$ group is not involved in the adsorption and is free as sketched in figure 5. This observation is very significant, as it is known to impart biocompatibility to Q-CdSe (Aldana et al 2001). In general, all the peaks compared to mercaptoethanol show a red shift compared to pristine mercaptoethanol (refer table 2). It can be interpreted as decrease in the bond order due to chemisorption.

\subsection{Photoluminescence (PL) measurements}

PL spectrum $\left(\lambda_{\text {exc }}=326 \mathrm{~nm}\right)$ recorded for redispersed QCdSe in DMSO is shown in figure 6 . The $\lambda_{\text {exc }}$ dependent sharp peak at $362 \mathrm{~nm}$, which showed $3050 \mathrm{~cm}^{-1}$ stokes shift relative to $\lambda_{\text {exc }}$ was attributed to the Raman scattering for DMSO. The broad peak at $457 \mathrm{~nm}$, more or less independent of $\lambda_{\text {exc }}$ is attributed to transition associated to the surface states in Q-CdSe. The red shift of ca. $131 \mathrm{~nm}$ from the absorption maxima was attributed to a dominant contribution from the surface states.

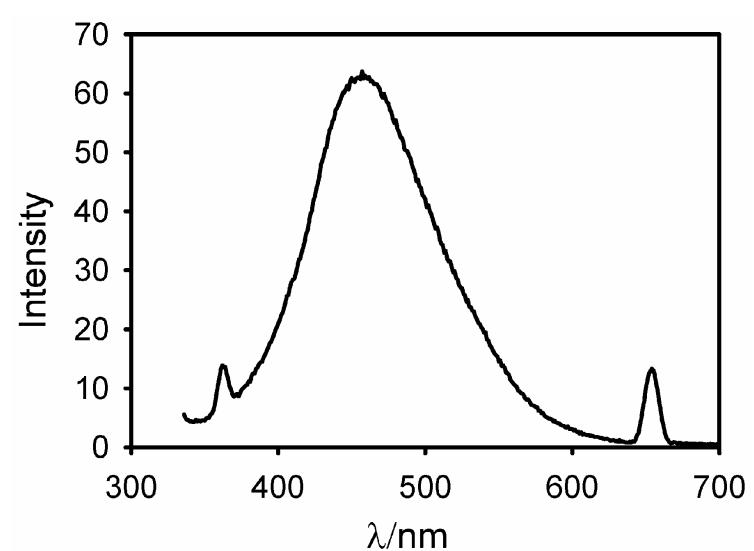

Figure 6. Photoluminescence spectrum recorded on Q-CdSe sol dispersed in DMSO. 
Table 2. FTIR analysis

\begin{tabular}{llll}
\hline Type of vibrations & Reported* $\left(\mathrm{cm}^{-1}\right)$ & $\begin{array}{c}\text { 2-Mercaptoethanol } \\
\left(\mathrm{cm}^{-1}\right)\end{array}$ & Q-CdSe $\left(\mathrm{cm}^{-1}\right)$ \\
\hline O-H stretching & 3300 & 3400 & 3367 \\
$\mathrm{C}-\mathrm{H}$ stretching, $\mathrm{CH}_{2}$ assym. & $2936-2916$ & 2943,2885 & 2930,2870 \\
$\mathrm{~S}-\mathrm{H}$ stretching & $2590-2540$ & 2563 & Absent \\
O-H deformation & $1360-1420$ & 1423 & 1421 \\
$\mathrm{CH}_{2}$ wagging & $1347-1182$ & 1296,1174 & 1286,1163 \\
Alcohol CH $_{2}-\mathrm{OH}, \mathrm{C}-\mathrm{O}$ primary stretching & $1075-1000$ & 1070 & 1063,1013 \\
$\mathrm{CH}_{2}$ rock & $1174-724$ & 948,912 & 943,835 \\
$\mathrm{C}-\mathrm{S}$ stretching & $705-570$ & 667 & 627 \\
\hline
\end{tabular}

*(Hostetler et al 1996; Zhang and Wang 2003).
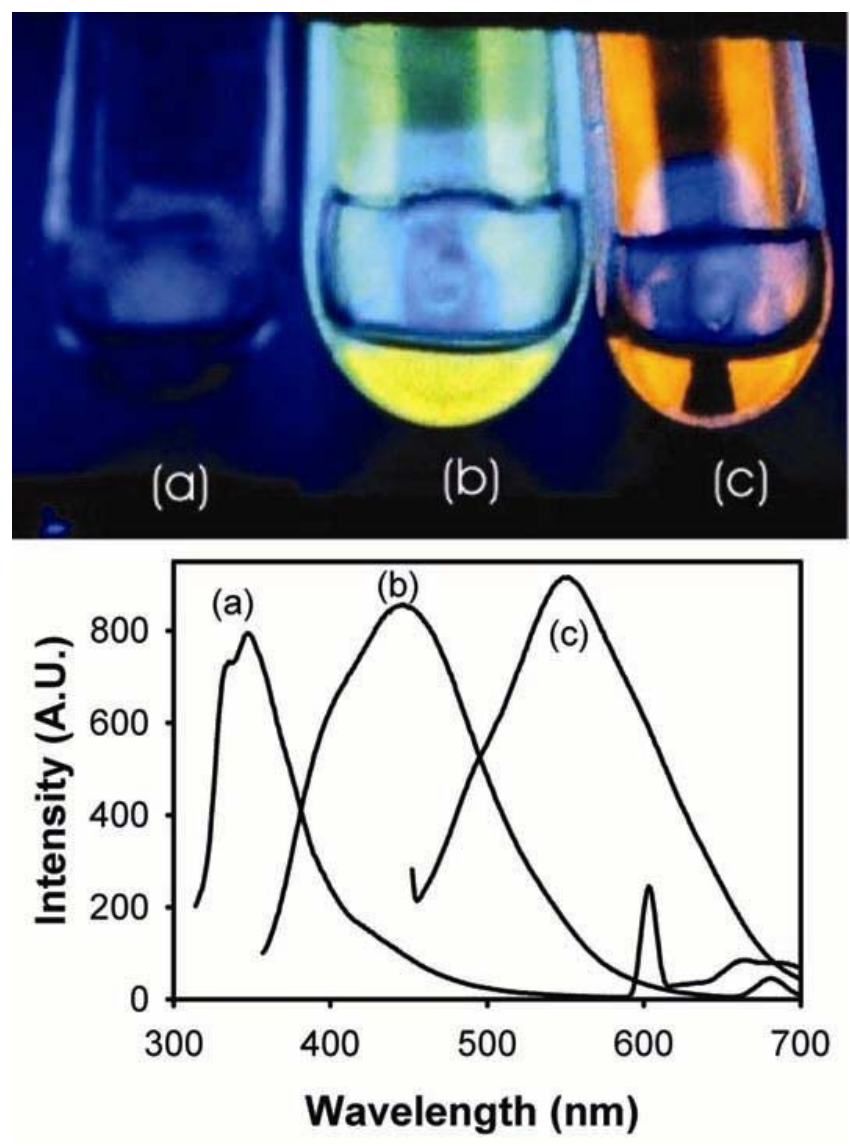

Figure 7. Photoluminescence spectrum recorded on Q-CdSe sol dispersed in DMSO

It is well documented in literature that the luminescence property of Q-CdSe can be varied by controlling the particle size (Dabbousi et al 1995; Trindade et al 2001; Vasiliev et al 2004). We achieved it by either separating various size fractions by a method of size selective precipitation or by changing the reaction conditions itself.

For example, PL recorded for the second and first fractions of one of the sols prepared is depicted in figures 7 (a) and (b), respectively. The second fraction was having smaller particle size, it displayed blue shift as compared to the first fraction. The spectrum in figure 7(c) was recorded for the sample in which the concentration of 2mercaptoethanol was half than that of the sample used for spectra shown in figures 7(a) and (b). On decreasing 2mercaptoethanol concentration, formation of bigger size particle was expected, which was manifested as red shift in the PL spectrum as seen in figure 7(c). Photographs of PL for all three samples obtained under illumination with $20 \mathrm{~mW}$ black UV light $(315-280 \mathrm{~nm}$ ) is shown in figure 7. Blue, green and orange PL were observed for these samples. Thus, we produced Q-CdSe sols, which gave PL in almost entire visible spectrum of light. To our knowledge, such clean and intense PL has been demonstrated only with TOPO capped Q-CdSe.

\section{Conclusions}

New single pot method has been developed for the synthesis of highly luminescent mercaptoethanoate capped Q-CdSe. It neither required a high temperature nor an inert atmosphere: (i) The solubility of these particles in the polar solvents viz. DMSO, DMF and their mixtures with water, (ii) presence of free $-\mathrm{OH}$ group on the particle available for the linking, and (iii) tunable luminescent properties make these particles a potential candidate for bio-labeling applications.

\section{References}

Aldana J, Wang Y A and Peng X $2001 \mathrm{~J}$. Am. Chem. Soc. 123 8844

Bowen-Katari J E, Colvin V L and Alivisatos A P 1994 J. Phys. Chem. 984109

Bruchez Jr M, Moronne M, Gin P, Weiss S and Alivisatos A P 1998 Science 2812013

Chan W C W and Nie S 1998 Science 2812016

Chen Y and Rosenzweig Z 2002 Anal. Chem. 745132

Chen Y, Ji T and Rosenzweig Z 2003 Nano Lett. 3581

Dabbousi B O, Bawendi M G, Onitsuka O and Rubner MF 1995 Appl. Phys. Lett. 661316 
Gaponik N, Talapin D V, Rogach A L, Hoppe K, Shevchenko E V, Kornowski A, Eychmu"ller A and Weller H $2002 \mathrm{~J}$. Phys. Chem. B106 7177

Gautam U K, Rajamathi M, Meldrum F, Morgan P and Seshadri R 2001 Chem. Commun. 629

Hostetler M J, Stokes J J and Murray R W 1996 Langmuir 12 3604

Hu J, Li L S, Yang W, Manna L, Wang L W and Alivisatos A P 2001 Science 2922060

Joint Committee on Powder Diffraction Standard, JCPDS File, $8-459$

Lin Y W, Hsieh M M, Liu C P and Chang H T 2005 Langmuir 21728

Mattoussi H, Mauro J M, Goldman E R, Green T M, Anderson G P, Sundar V C and Bawendi M G 2001 Phys. Status Solidi B224 277

Ma Xiao-Dong, Qian Xue-Feng, Yin Jie, Xi Hong-An and Zhu Z K 2002 J. Colloid \& Interface Sci. 25277

Murray C B, Norris D J and Bawendi M G 1993 J. Am. Chem. Soc. 1158706
Parak W J et al 2002 Chem. Mater. 142113

Pathak S, Choi S K, Arnheim N and Thompson ME $2001 \mathrm{~J}$. Am. Chem. Soc. 1234103

Peng Z A and Peng X 2001 J. Am. Chem. Soc. 123183

Rogach A L, Kornowski A, Gao M, Eychmu"ller A and Weller H 1999 J. Phys. Chem. B103 3065

Rogach A L, Nagesha D, Ostrander J W, Giersig M and Kotov N A 2000 Chem. Mater. 122676

Tian Y, Newton T, Kotov N A, Guldi D M and Fendler J H 1996 J. Phys. Chem. 1008927

Trindade T and O'Brien P 1996 Adv. Mater. 8161

Trindade T, O'Brien P and Zhang X 1997 Chem. Mater. 9 523

Trindade T, O'Brien P and Pickett N L 2001 Chem. Mater. 13 3843

Vasiliev R B, Dorofeev S G, Dirin D N, Belov D A and Kuznetsova T A 2004 Mendeleev Commun. 169

Xie Y, Wang W Z, Qian Y T and Liu X M 1999 J. Solid State Chem. 4782

Zhang X and Wang S 2003 Sensors 361 